\title{
Open-system quantum dynamics with correlated initial states, not completely positive maps, and non-Markovianity
}

\author{
A. R. Usha Devi, ${ }^{1,2,{ }^{*}}$ A. K. Rajagopal, ${ }^{2}$ and Sudha ${ }^{3,4}$ \\ ${ }^{1}$ Department of Physics, Bangalore University, Bangalore-560 056, India \\ ${ }^{2}$ Inspire Institute Inc., Alexandria, Virginia 22303, USA \\ ${ }^{3}$ Department of Physics, Kuvempu University, Shankaraghatta, Shimoga-577 451, India \\ ${ }^{4}$ DAMTP, Centre for Mathematical Sciences, Wilberforce Road, Cambridge CB3 OWA, UK
}

(Received 27 October 2010; published 28 February 2011)

\begin{abstract}
Dynamical $A$ and $B$ maps have been employed extensively by Sudarshan and co-workers to investigate open-system evolution of quantum systems. A canonical structure of the $A$ map is introduced here. It is shown that this canonical $A$ map enables us to investigate whether the dynamics is completely positive (CP) or not completely positive (NCP) in an elegant way and, hence, it subsumes the basic results on open-system dynamics. Identifying memory effects in open-system evolution is gaining increasing importance recently and, here, a criterion of non-Markovianity, based on the relative entropy of the dynamical state is proposed. The relative entropy difference of the dynamical system serves as a complementary characterization-though not related directly - to the fidelity difference criterion proposed recently. Three typical examples of open-system evolution of a qubit, prepared initially in a correlated state with another qubit (environment), and evolving jointly under a specific unitary dynamics—which corresponds to a NCP dynamical map—are investigated by employing both the relative entropy difference and fidelity difference tests of non-Markovianity. The two-qubit initial states are chosen to be (i) a pure entangled state, (ii) the Werner state, which exemplifies both entangled and separable states of qubits, depending on a real parameter, and (iii) a separable mixed state. Both the relative entropy and fidelity criteria offer a nice display of how non-Markovianity manifests itself in all three examples.
\end{abstract}

DOI: 10.1103/PhysRevA.83.022109

PACS number(s): 03.65.Yz, 03.65.Ta, 42.50.Lc

\section{INTRODUCTION}

It is well known [1] that when a quantum system-chosen initially to be in a tensor product state with its environmental degrees of freedom - undergoes dynamical evolution, the final state of the system is related to the initial one via a completely positive (CP) dynamical map. Kraus decomposition [2] of dynamics is guaranteed only when the map is completely positive. After its conceptual formulation [3,4] nearly five decades ago, Sudarshan and coworkers [5-8] have been investigating the quantum theory of open-system evolution in terms of dynamical maps in the more general settingincluding not completely positive (NCP) maps. There has been growing interest [9-13] in identifying the physical conditions under which open evolution of a quantum system does not ensure a CP dynamical map. Jordan et al. [5] studied an open-system unitary evolution, where the system and the environment may be in an initially entangled state, and showed that the resulting dynamical map is not always completely positive. Rodríguez-Rosario and Sudarshan [7] analyzed the general characteristics of dynamical maps in open quantum system evolutions, taking into account initial correlations of the system with its environment. Extending the result of Ref. [6], Shabani and Lidar [13] showed recently that CP maps are guaranteed for quantum dynamical processes, if and only if the initial system-environment state belongs to a class of separable states with vanishing quantum discord [14]. All these investigations point toward the important role of the initial state in open-system evolution and more recently, Modi

*arutth@rediffmail.com and Sudarshan [8] outlined the effects of preparation of the initial state in quantum process tomography.

The Markov approximation, where the correlation time between the system and environment is considered to be infinitesimally small—so that the dynamical map does not carry any memory effects-leads to a much simplified picture of opensystem dynamics. The mathematical theory of Markovian dynamics is built around a CP map_originating from the unitary evolution of an initially uncorrelated system-environment state and generating a dynamical semigroup (to take into account the memorylessness). This results in the Markovian dynamical equation-known as the Lindblad-Gorini-KossakowskiSudarshan (LGKS) [15,16] master equation-for the time evolution of the system density matrix. However, memory effects are prevalent in many physical situations of interest. Formulation of CP non-Markovian processes-where the dynamical evolution depends on the history of the systemenvironment correlation-has attracted significant attention [7,17-19]. Various manifestations of non-Markovianity have been investigated recently [20-23], based on the departure from strict Markovian behavior (where the dynamical map is a one-parameter continuous, memoryless, completely positive semigroup). However, revelation of non-Markovian features under NCP maps has not been studied so far. In the present paper, we focus on two basic issues: (1) a canonical structure of the dynamical map to elucidate $\mathrm{CP}$ or NCP nature of dynamics and (2) operational signatures of non-Markovianity. We consider a particular unitary time evolution-which has been shown [5] to correspond to a NCP map - and examine the non-Markovianity of the open-system dynamics of singlequbit systems, resulting from the joint evolution of different types of initially correlated two-qubit states. We propose a 
criterion, based on the relative entropy of the quantum states, to verify Markovianity and non-Markovianity of the dynamical process. We compare the results with the fidelity difference [23] test of non-Markovianity proposed recently by some of us [23].

We first review the properties of $A$ and $B$ maps [3,6], introduced in the general theory of open-system dynamics in Sec. II. Here, we present a new canonical structure of the $A$ map, which reveals its equivalence with that of the $B$ map. We employ this canonical map to establish $\mathrm{CP}$ or NCP nature of the dynamics. In Sec. III we explore a specific example of open-system unitary evolution [5] of a single qubit (system), which is initially prepared in a correlated state with another qubit (environment), leading to a NCP dynamical map. This map serves as a template for studying the different examples of initially correlated two-qubit states considered here. We construct a characterization of nonMarkovianity in Sec. IV, based on relative entropy of the evolving quantum states. We also discuss the fidelity difference criterion [23] of non-Markovianity proposed earlier so as to be self-contained and mutually concordant. We then proceed to illustrate non-Markovian behavior of the NCP dynamical map of Sec. III, with the help of three different choices of density matrices prepared initially in entangled pure, mixed, and separable states of the two qubits. By employing the relative entropy criterion, as well as the fidelity difference test, we show that the dynamics in all three different cases exhibits non-Markovianity. Section IV is devoted to a brief summary of our results.

\section{REVIEW ON THE GENERAL PROPERTIES OF $A$ AND $B$ DYNAMICAL MAPS AND THEIR EQUIVALENCE}

A general open-system dynamics relates the elements $[\rho(0)]_{r s}$ of the initial system density matrix with $[\rho(t)]_{r^{\prime} s^{\prime}}$ at instant $t$ via a linear map [3,6],

$$
[\rho(t)]_{r^{\prime} s^{\prime}}=\sum_{r, s=1}^{n} A_{r^{\prime} s^{\prime} ; r s}(t)[\rho(0)]_{r s}, \quad r^{\prime}, s^{\prime}=1,2, \ldots, n .
$$

Imposing that the $A$ map ensures (i) the preservation of hermiticity, i.e., $[\rho(t)]_{r^{\prime} s^{\prime}}=[\rho(t)]_{s^{\prime} r^{\prime}}^{*}$, and (ii) a unit trace condition, i.e., $\operatorname{Tr}[\rho(t)]=1$, the following restrictions on the elements of $A$ are realized [3,6]:

$$
\begin{aligned}
& A_{s^{\prime} r^{\prime} ; s r}=A_{r^{\prime} s^{\prime} ; r s}^{*}, \\
& \sum_{r^{\prime}} A_{r^{\prime} r^{\prime} ; r s}=\delta_{r, s} .
\end{aligned}
$$

In order to bring out the properties (2) and (3) in a transparent manner, it has been found convenient to define a realigned matrix $B[3,6]$ :

$$
B_{r^{\prime} r ; s^{\prime} s}=A_{r^{\prime} s^{\prime} ; r s} .
$$

The hermiticity property (2) leads to the condition $B_{s^{\prime} s ; r^{\prime} r}=$ $B_{r^{\prime} r ; s^{\prime} s}^{*}$, i.e., the dynamical map $B$ itself is hermitian-which is exploited further to identify the general features of dynamics $[3,6]$. Here, we present an alternate version by casting the $A$ map in its canonical form, which is shown to capture all the dynamical features in an unique way. To accomplish this, we start by considering an orthonormal set $\left\{T_{\alpha}, \alpha=1,2, \ldots, n^{2}\right\}$ of $n \times n$ basis matrices, satisfying

$$
\operatorname{Tr}\left[T_{\alpha}^{\dagger} T_{\beta}\right]=\delta_{\alpha, \beta},
$$

so that we can express the $n^{2} \times n^{2}$ matrix $A$ as

$$
\begin{gathered}
A=\sum_{\alpha \beta} \mathcal{A}_{\alpha \beta} T_{\alpha} \otimes T_{\beta}^{*}, \\
\mathcal{A}_{\alpha \beta}=\operatorname{Tr}\left[A\left(T_{\alpha}^{\dagger} \otimes T_{\beta}^{T}\right)\right] .
\end{gathered}
$$

Clearly, we have

$$
A_{r^{\prime} s^{\prime} ; r s}=\sum_{\alpha, \beta=1}^{n^{2}} \mathcal{A}_{\alpha \beta}\left[T_{\alpha}\right]_{r^{\prime} r}\left[T_{\beta}^{*}\right]_{s^{\prime} s}
$$

The hermiticity preservation condition (2) implies that

$$
\mathcal{A}_{\alpha \beta}=\mathcal{A}_{\beta \alpha}^{*}
$$

i.e., the coefficients $\mathcal{A}_{\alpha \beta}$ form an $n^{2} \times n^{2}$ hermitian matrix $\mathcal{A}$. Denoting $\mathcal{U}$ as the matrix diagonalizing $\mathcal{A}$ and $\left\{\lambda_{\mu}\right\}$ as the real eigenvalues of $\mathcal{A}$, so that $\sum_{\alpha, \beta} \mathcal{U}_{\mu \alpha} \mathcal{A}_{\alpha \beta} \mathcal{U}_{\mu \beta}^{*}=\lambda_{\mu}$, we finally obtain the canonical structure of the $A$ map:

$$
A=\sum_{\alpha, \beta, \mu} \lambda_{\mu} \mathcal{U}_{\mu \beta} \mathcal{U}_{\mu \alpha}^{*} T_{\alpha} \otimes T_{\beta}^{*}=\sum_{\mu} \lambda_{\mu} \mathcal{C}_{\mu} \otimes \mathcal{C}_{\mu}^{*},
$$

where $\mathcal{C}_{\mu}=\sum_{\alpha} \mathcal{U}_{\mu \alpha}^{*} T_{\alpha}$. With the help of the canonical form, the matrix elements of $A$ are explicitly given by

$$
A_{r^{\prime} s^{\prime} ; r s}=\sum_{\mu} \lambda_{\mu}\left[\mathcal{C}_{\mu}\right]_{r^{\prime} r}\left[\mathcal{C}_{\mu}^{*}\right]_{s^{\prime} s}
$$

Substituting (11) in (1) and simplifying, we obtain the following elegant form for the action of the $A$ map on the initial density matrix $\rho(0)$ :

$$
\rho(t)=\sum_{\mu} \lambda_{\mu} \mathcal{C}_{\mu} \rho(0) \mathcal{C}_{\mu}^{\dagger} .
$$

The trace preservation condition (3) can be readily expressed as $\sum_{\mu} \lambda_{\mu} \mathcal{C}_{\mu}^{\dagger} \mathcal{C}_{\mu}=I$ (where $I$ denotes the $n \times n$ identity matrix). The dynamical map is CP, when all the eigenvalues $\lambda_{\mu}$ are non-negative, whereas it is NCP if at least one of them is negative [24].

From (4) and (11) we obtain, $B_{r^{\prime} r ; s^{\prime} s}=\sum_{\mu} \lambda_{\mu}\left[\mathcal{C}_{\mu}\right]_{r^{\prime} r}$ $\left[\mathcal{C}_{\mu}^{*}\right]_{s^{\prime} s}$, which evidently corresponds to the spectral decomposition of the $B$ matrix. In other words, the eigenvalues of the matrices $\mathcal{A}$ and $B$ are identically the same and $\left[\mathcal{C}_{\mu}\right]_{r^{\prime} r}$ (expressed as an $n^{2}$ component column) correspond to the corresponding eigenvectors of $B$. In this paper we employ the canonical form of the $A$ map to elucidate the $\mathrm{CP}$ or NCP nature of the evolution.

\section{AN EXAMPLE OF TWO-QUBIT UNITARY DYNAMICS}

Jordan et al. [5] studied a specific example of unitary dynamical evolution on two-qubit states $U(t)=e^{-i H t / \hbar}$, governed by the Hamiltonian

$$
H=\frac{1}{2} \hbar \omega \sigma_{1 z} \sigma_{2 x},
$$

where $\sigma_{1 i}$ and $\sigma_{2 i}, i=x, y, z$, respectively, denote Pauli matrices of first and second qubits. The unitary tranformation 
matrix on the qubits is given explicitly (in the standard qubit basis $|0,0\rangle,|0,1\rangle,|1,0\rangle,|1,1\rangle)$ by

$$
U(t)=\left(\begin{array}{cccc}
\cos \left(\frac{\omega t}{2}\right) & -i \sin \left(\frac{\omega t}{2}\right) & 0 & 0 \\
-i \sin \left(\frac{\omega t}{2}\right) & \cos \left(\frac{\omega t}{2}\right) & 0 & 0 \\
0 & 0 & \cos \left(\frac{\omega t}{2}\right) & i \sin \left(\frac{\omega t}{2}\right) \\
0 & 0 & i \sin \left(\frac{\omega t}{2}\right) & \cos \left(\frac{\omega t}{2}\right)
\end{array}\right) .
$$

The positive definiteness of the dynamical state of the system qubit—evolving jointly with another environment qubit under the unitary time evolution (14) - is necessarily preserved.

Time evolution of the expectation values of the Pauli operators of the first qubit—evaluated in the Heisenberg picture-are given by

$$
\begin{aligned}
&\left\langle U^{\dagger}(t) \sigma_{1 x} U(t)\right\rangle=\left\langle\sigma_{1 x}\right\rangle \cos (\omega t)-\left\langle\sigma_{1 y} \sigma_{2 x}\right\rangle \sin (\omega t) \\
&=\left\langle\sigma_{1 x}\right\rangle \cos (\omega t)+a_{1} \sin (\omega t), \\
&\left\langle U^{\dagger}(t) \sigma_{1 y} U(t)\right\rangle=\left\langle\sigma_{1 y}\right\rangle \cos (\omega t)+\left\langle\sigma_{1 x} \sigma_{2 x}\right\rangle \sin (\omega t) \\
&=\left\langle\sigma_{1 y}\right\rangle \cos (\omega t)+a_{2} \sin (\omega t), \\
&\left\langle U^{\dagger}(t) \sigma_{1 z} U(t)\right\rangle=\left\langle\sigma_{1 z}\right\rangle,
\end{aligned}
$$

where $\left\langle\sigma_{1 x}\right\rangle,\left\langle\sigma_{1 y}\right\rangle$, and $\left\langle\sigma_{1 z}\right\rangle$ are the expectation values at $t=0$ and

$$
a_{1}=-\left\langle\sigma_{1 y} \sigma_{2 x}\right\rangle, a_{2}=\left\langle\sigma_{1 x} \sigma_{2 x}\right\rangle
$$

are considered to be the fixed initial-state parameters describing the evolution of the first qubit [5]. Correspondingly, the density matrix of the first qubit,

$$
\rho_{1}(0)=\frac{1}{2}\left(I_{1}+\sigma_{1 x}\left\langle\sigma_{1 x}\right\rangle+\sigma_{1 y}\left\langle\sigma_{1 y}\right\rangle+\sigma_{1 z}\left\langle\sigma_{1 z}\right\rangle\right),
$$

is mapped to

$$
\begin{aligned}
\rho_{1}(t)= & \frac{1}{2}\left[I_{1}+\left(a_{1} \sigma_{1 x}+a_{2} \sigma_{1 y}\right) \sin (\omega t)+\sigma_{1 x}\left\langle\sigma_{1 x}\right\rangle \cos (\omega t)\right. \\
& \left.+\sigma_{1 y}\left\langle\sigma_{1 y}\right\rangle \cos (\omega t)+\sigma_{1 z}\left\langle\sigma_{1 z}\right\rangle\right] .
\end{aligned}
$$

Equation (20) in turn corresponds to [5]

$$
\begin{gathered}
I_{1}^{\prime}=I_{1}+\left(a_{1} \sigma_{1 x}+a_{2} \sigma_{2 x}\right) \sin (\omega t), \\
\sigma_{1 x}^{\prime}=\sigma_{1 x} \cos (\omega t), \\
\sigma_{1 y}^{\prime}=\sigma_{1 y} \cos (\omega t), \\
\sigma_{1 z}^{\prime}=\sigma_{1 z} .
\end{gathered}
$$

For fixed parameters $a_{1}$ and $a_{2}$ characterizing the initial state, a linear dynamical $A$ map $Q \rightarrow Q^{\prime}$ for all $2 \times 2$ hermitian matrices - consistent with the unitary evolution (14) - is defined by [see Eq. (1)]

$$
Q_{r s}^{\prime}=\sum_{r^{\prime} s^{\prime}} A_{r s ; r^{\prime} s^{\prime}} Q_{r^{\prime} s^{\prime}}, r, s, r^{\prime}, s^{\prime}=0,1,
$$

where

$$
A=\left(\begin{array}{cccc}
1 & 0 & 0 & 0 \\
\frac{1}{2} S a^{*} & C & 0 & \frac{1}{2} S a^{*} \\
\frac{1}{2} S a & 0 & C & \frac{1}{2} S a \\
0 & 0 & 0 & 1
\end{array}\right)
$$

with $a=a_{1}+i a_{2}, C=\cos (\omega t)$, and $S=\sin (\omega t)$.
Choosing $\left\{\frac{\sigma_{1 \alpha}}{\sqrt{2}} \equiv \frac{I_{1}}{\sqrt{2}}, \frac{\sigma_{1 x}}{\sqrt{2}}, \frac{\sigma_{1 y}}{\sqrt{2}}, \frac{\sigma_{1 z}}{\sqrt{2}}\right\}$ as the orthonormal set of basis matrices, we expand the $A$ matrix (23) as [see Eq. (6)]

$$
\begin{aligned}
& A=\frac{1}{2} \sum_{\alpha \beta} \mathcal{A}_{\alpha \beta} \sigma_{\alpha} \otimes \sigma_{\beta}^{*}, \\
& \mathcal{A}_{\alpha \beta}=\frac{1}{2} \operatorname{Tr}\left[A\left(\sigma_{\alpha} \otimes \sigma_{\beta}^{*}\right)\right],
\end{aligned}
$$

with the hermitian coefficient matrix $\mathcal{A}$ given by

$$
\begin{aligned}
\mathcal{A} & =\frac{1}{2} \operatorname{Tr}\left[A(t) \sigma_{\alpha} \otimes \sigma_{\beta}^{*}\right] \\
& =\frac{1}{2}\left(\begin{array}{cccc}
2(1+C) & a_{1} S & a_{2} S & 0 \\
a_{1} S & 0 & 0 & i a_{2} S \\
a_{2} S & 0 & 0 & -i a_{1} S \\
0 & -i a_{2} S & i a_{1} S & 2(1-C)
\end{array}\right) .
\end{aligned}
$$

The eigenvalues of $\mathcal{A}$ are given by [25]

$$
\begin{aligned}
& \lambda_{1 \pm}=\frac{1}{2}\left\{[1+\cos (\omega t)] \pm \sqrt{[1+\cos (\omega t)]^{2}+|a|^{2} \sin ^{2}(\omega t)}\right\}, \\
& \lambda_{2 \pm}=\frac{1}{2}\left\{[1-\cos (\omega t)] \pm \sqrt{[1-\cos (\omega t)]^{2}+|a|^{2} \sin ^{2}(\omega t)}\right\} .
\end{aligned}
$$

It may be seen that $\lambda_{1-}$ and $\lambda_{2-}$ assume negative values and thus the dynamical map is NCP [5] (see however [26]). Nevertheless, as pointed out earlier, the positive definiteness of the dynamical single-qubit state, evolving jointly under the unitary dynamics (14) with another qubit (which are prepared in an initially correlated state) is always ensured. The $A$ map (23) serves as a general dynamical map for the different examples considered in Sec. V.

\section{NON-MARKOVIAN FEATURES}

We recall here that an open-system CP dynamical map is Markovian if it forms a one-parameter semigroup [1], which corresponds to

$$
A(t+\tau)=A(t) A(\tau), \quad t, \tau \geqslant 0,
$$

for the $A$ map (1). In other words, when the underlying $\mathrm{CP}$ dynamics is Markovian, the $A$ map has an exponential structure $A=e^{t L}$, with $L$ denoting the time-independent generator of the quantum dynamical semigroup $[15,16]$. One may verify directly whether the one-parameter semigroup criterion [Eq. (28)] is obeyed by checking if the $A$ map is exponential. However, it is advantageous to examine physical quantities, which are functions of the dynamical map, that describe the open-system evolution of the physical states. In the following we consider the relative entropy difference and the fidelity difference to qualitatively capture the departure from the CP Markovian semigroup property of evolution.

We consider here the relative entropy [27] of two density matrices $\rho$ and $\gamma$, defined by

$$
S(\rho \| \gamma)=\operatorname{Tr}[\rho(\ln \rho-\ln \gamma)],
$$

which is positive and vanishes if and only if $\rho \equiv \gamma$. Under $\mathrm{CP}$, trace-preserving dynamical maps $\Phi$, the relative entropy obeys the monotonicity property [28], i.e.,

$$
S[\Phi(\rho) \| \Phi(\gamma)] \leqslant S(\rho \| \gamma) .
$$


Thus, it follows that

$$
\begin{aligned}
S[\rho(t) \| \rho(t+\tau)] & \equiv S[A(t) \rho(0) \| A(t) \rho(\tau)] \\
& \leqslant S[\rho(0) \| \rho(\tau)]
\end{aligned}
$$

under a trace-preserving $\mathrm{CP}$ map $A: \rho(0) \rightarrow \rho(t)=$ $A(t) \rho(0)$, obeying the Markovian semigroup property (28). In other words, the relative entropy difference defined as

$$
S(t, \tau)=S[\rho(0) \| \rho(\tau)]-S[\rho(t) \| \rho(t+\tau)]
$$

is necessarily positive for all quantum states $\rho(t)$ evolving under CP Markovian dynamics. The inequality (31) need not be satisfied by both NCP processes as well as by a CP evolution, which departs from the semigroup property (28). Thus, violation of the inequality (31), i.e.,

$$
S(t, \tau)<0
$$

signifies a non-Markovian dynamical process (both CP as well as $\mathrm{NCP}$ ).

We also recall here that the fidelity function [29] defined by

$$
F[\rho(t), \rho(t+\tau)]=\{\operatorname{Tr}[\sqrt{\sqrt{\rho(t)} \rho(t+\tau) \sqrt{\rho(t)}}]\}^{2},
$$

never decreases from its initial value $F[\rho(0), \rho(\tau)]$ [23] for the state $\rho(t)$ undergoing a Markovian CP dynamical process. A sufficient criterion for non-Markovianity is therefore registered-if the fidelity difference function [23]

$$
G(t, \tau)=\frac{F[\rho(t), \rho(t+\tau)]-F[\rho(0), \rho(\tau)]}{F[\rho(0), \rho(\tau)]}
$$

assumes negative values under open-system dynamics.

The relative entropy and fidelity exhibit contrasting physical implications: The relative entropy $S[\rho(t) \| \rho(t+\tau)]$ measures the instantaneous distinguishability of the dynamical state $\rho(t+\tau)$ with its earlier time density matrix $\rho(t)$ and it declines-when the system undergoes a CP Markovian process-from its initial value $S[\rho(0) \| \rho(\tau)]$ to its minimum value asymptotically, i.e., $\lim _{t \rightarrow \infty} S[\rho(t) \| \rho(t+\tau)]=$ 0 . On the other hand, the fidelity $F[\rho(t), \rho(t+\tau)]$ signifies the overlap of the dynamical states $\rho(t+\tau), \rho(t)$; under any CP Markovian dynamics, it increases monotonically from its initial value $F[\rho(0), \rho(\tau)]$ to its maximum value $\lim _{t \rightarrow \infty} F[\rho(t), \rho(t+\tau)]=1$.

The negative values of relative entropy difference (32) and the fidelity difference (35) point out that the time evolution is not a CP Markovian process-though their positive values do not necessarily suggest that the dynamics is Markovian. In other words, the negative values of relative entropy and fidelity differences serve as sufficient-but not necessarytests of non-Markovianity (CP as well as NCP). Further, it is not possible to draw any clear-cut inference toward whether the open-system dynamics is NCP or not-based entirely on the negative values of the quantities (32) and (35). However, these signatures of non-Markovianity, in terms of relative entropy and fidelity, offer an operational advantage that they require only the specification of the initial density matrix $\rho(0)$, and the dynamically evolved one $\rho(t)$ for their evaluation-without any a priori knowledge on the nature of the environment and/or the coupling between the system and environment.

\section{ILLUSTRATIVE EXAMPLES}

We now proceed to investigate three different examples of two-qubit initial states, jointly undergoing the unitary transformation (14) so that the time evolution of the first qubit (system) is represented by the dynamical map

$$
\rho_{1}(0) \rightarrow \rho_{1}(t)=A \rho_{1}(0)=\operatorname{Tr}_{2}\left[U(t) \rho_{12}(0) U(t)^{\dagger}\right] .
$$

The examples are indeed expected to reveal non-Markovian features as the environment consists of just a single qubit, with no additional assumptions on the weak-coupling limit [1] invoked (so as to lead to CP Markovian dynamics, when initially uncorrelated states are considered [26]). Further, as the dynamical evolution of the system qubit is governed by the NCP dynamical map (23), non-Markovianity is bound to emerge. Here, we focus on the non-Markovian features by verifying that the relative entropy difference (32) and the fidelity difference (35) assume negative values under this open-system NCP dynamics.

\section{A. Example 1: Pure entangled two-qubit state}

We first consider a two-qubit pure entangled state

$$
\left|\Psi_{\mathrm{EP}}\right\rangle=\frac{1}{\sqrt{3}}\left(e^{-i \phi}\left|0_{1}, 1_{2}\right\rangle+e^{i \phi}\left|1_{1}, 0_{2}\right\rangle+\left|1_{1}, 1_{2}\right\rangle\right),
$$

with the corresponding initial density matrix of the system qubit given by

$$
\rho_{1}(t=0)=\frac{1}{3}\left(\begin{array}{cc}
1 & e^{-i \phi} \\
e^{i \phi} & 2
\end{array}\right)
$$

in the standard basis $\{|0\rangle,|1\rangle\}$.

Under the unitary transformation (14) we explicitly obtain the dynamical state of the system qubit as

$$
\begin{aligned}
\rho_{1}(t) & =\operatorname{Tr}_{2}\left[U(t)\left|\Psi_{\mathrm{EP}}\right\rangle\left\langle\Psi_{\mathrm{EP}}\right| U^{\dagger}(t)\right] \\
& =\frac{1}{3}\left(\begin{array}{cc}
1 & C e^{-i \phi}-i S e^{-2 i \phi} \\
C e^{i \phi}+i S e^{2 i \phi} & 2
\end{array}\right) .
\end{aligned}
$$

This may also be identified to be the result of the action of the open-system dynamical $A$ map (23):

$$
\begin{aligned}
& \frac{1}{3}\left(\begin{array}{c}
1 \\
C e^{-i \phi}-i S e^{-2 i \phi} \\
C e^{i \phi}+i S e^{2 i \phi} \\
2
\end{array}\right) \\
& =\frac{1}{3}\left(\begin{array}{cccc}
1 & 0 & 0 & 0 \\
\frac{1}{2} S a^{*} & C & 0 & \frac{1}{2} S a^{*} \\
\frac{1}{2} S a & 0 & C & \frac{1}{2} S a \\
0 & 0 & 0 & 1
\end{array}\right)\left(\begin{array}{c}
1 \\
e^{-i \phi} \\
e^{i \phi} \\
2
\end{array}\right)
\end{aligned}
$$

with the initial state parameters [see (18)]

$$
\begin{gathered}
a_{1}=-\left\langle\Psi_{\mathrm{EP}}\left|\sigma_{1 y} \sigma_{2 x}\right| \Psi_{E P}\right\rangle=-\frac{2}{3} \sin (2 \phi), \\
a_{2}=\left\langle\Psi_{\mathrm{EP}}\left|\sigma_{1 x} \sigma_{2 x}\right| \Psi_{E P}\right\rangle=\frac{2}{3} \cos (2 \phi)
\end{gathered}
$$

governing the open-system dynamics. 
We obtain, after simplification, the relative entropy $S\left[\rho_{1}(t) \| \rho_{1}(t+\tau)\right]$ of the dynamical state (39) of the qubit as

$$
\begin{aligned}
& S\left[\rho_{1}(t) \| \rho_{1}(t+\tau)\right] \\
& =\Lambda^{(+)}(t) \ln \left\{\frac{\Lambda^{(+)}(t)}{\left[\Lambda^{(+)}(t+\tau)\right]^{\delta(t)}\left[\Lambda^{(-)}(t+\tau)\right]^{\nu(t)}}\right\} \\
& \quad+\Lambda^{(-)}(t) \ln \left\{\frac{\Lambda^{(-)}(t)}{\left[\Lambda^{(+)}(t+\tau)\right]^{\nu(t)}\left[\Lambda^{(-)}(t+\tau)\right]^{\delta(t)}}\right\},
\end{aligned}
$$

where we have denoted

$$
\begin{aligned}
\Lambda^{( \pm)}(t)= & \frac{3 \pm \kappa(t)}{6}, \quad \kappa(t)=\sqrt{5-4 \sin (2 \omega t) \sin \phi}, \\
\delta(t)= & \frac{1}{2 \kappa(t) \kappa(t+\tau)}([\kappa(t) \kappa(t+\tau)+1] \\
& +4\{\cos (\omega \tau)-\sin [\omega(2 t+\tau)] \sin \phi\}), \\
v(t)= & \frac{1}{2 \kappa(t) \kappa(t+\tau)}([\kappa(t)+1][\kappa(t+\tau)-1] \\
& -4\{\cos (\omega \tau)-\sin [\omega(2 t+\tau)] \sin \phi\}) .
\end{aligned}
$$

In order to compute the fidelity $F\left[\rho_{1}(t), \rho_{1}(t+\tau)\right]$, we make use of its simplified form in the case of single-qubit states [29]:

$$
\begin{aligned}
F\left[\rho_{1}(t), \rho_{1}(t+\tau)\right]= & \operatorname{Tr}\left[\rho_{1}(t) \rho_{1}(t+\tau)\right] \\
& +2 \sqrt{\operatorname{det} \rho_{1}(t) \operatorname{det} \rho_{1}(t+\tau)} .
\end{aligned}
$$

We obtain the fidelity $F\left[\rho_{1}(t), \rho_{1}(t+\tau)\right]$ of the dynamical state (39) as

$$
\begin{aligned}
& F\left[\rho_{1}(t), \rho_{1}(t+\tau)\right] \\
& =\frac{1}{9}\{5+2 \cos (\omega \tau)-2 \sin [\omega(2 t+\tau)] \sin \phi \\
& \quad+2 \sqrt{[1+\sin (2 \omega t) \sin \phi]\{1+\sin [2 \omega(t+\tau)] \sin \phi\}}\} .
\end{aligned}
$$

The relative entropy difference $S(t, \tau)$ and the fidelity difference $G(t, \tau)$ [see (35)] for the dynamical state (39) are plotted as a function of $\omega t$ and $\phi$ in Fig. 1-where the negative regions of both $S(t, \tau), G(t, \tau)$ display the non-Markovianity of the dynamical process.

\section{B. Example 2: Two-qubit Werner state}

We consider Werner state of two qubits as the initial systemenvironment state

$$
\rho_{W}(t=0)=\frac{x}{4} I_{1} \otimes I_{2}+(1-x)\left|\Psi_{-}\right\rangle\left\langle\Psi_{-}\right|,
$$

where $\left.\left|\Psi_{-}\right\rangle=\frac{1}{\sqrt{2}}\left(\left|0_{1}, 1_{2}\right\rangle-\left|1_{1}, 0_{2}\right|\right\rangle\right)$. The initial state of the system qubit is given by $\rho_{W 1}(0)=\operatorname{Tr}_{2}\left[\rho_{W}(0)\right]=\frac{1}{2} I_{1}$.

Under open-system dynamics (14) we obtain the dynamical state of the system qubit as

$$
\begin{aligned}
\rho_{W 1}(t) & =\operatorname{Tr}_{2}\left[U(t) \rho_{W}(0) U^{\dagger}(t)\right] \\
& =\frac{1}{2}\left(\begin{array}{cc}
1 & -i(1-x) \sin (\omega t) \\
i(1-x) \sin (\omega t) & 1
\end{array}\right)
\end{aligned}
$$

(which may also be directly obtained by employing the $A$ map (23) as $\left[\rho_{W 1}(t)\right]_{r^{\prime} s^{\prime}}=\sum_{r, s} A_{r^{\prime} s^{\prime} ; r s}(t)\left[\rho_{W 1}(0)\right]_{r s}$ with $a_{1}=-\operatorname{Tr}\left[\rho_{W}(0) \sigma_{1} y \sigma_{2 x}\right]=0$ and $a_{2}=\operatorname{Tr}\left[\rho_{W}(0) \sigma_{1 x} \sigma_{2 x}\right]=$ $(1-x))$.

The relative entropy $S\left[\rho_{W 1}(t) \| \rho_{W 1}(t+\tau)\right]$ of the system qubit (47) is identified to be

$$
\begin{aligned}
S\left[\rho_{W 1}(t) \| \rho_{W 1}(t+\tau)\right]= & p_{+}(t) \ln \left[\frac{p_{+}(t)}{p_{+}(t+\tau)}\right] \\
& +p_{-}(t) \ln \left[\frac{p_{-}(t)}{p_{-}(t+\tau)}\right],
\end{aligned}
$$

where

$$
p_{ \pm}(t)=\frac{1}{2}[1 \pm(1-x) \sin (\omega t)] .
$$

Further, the fidelity $F\left[\rho_{W 1}(t), \rho_{W 1}(t+\tau)\right]$ is obtained as

$$
\begin{aligned}
& F\left[\rho_{W 1}(t), \rho_{W 1}(t+\tau)\right] \\
& =p_{+}(t) p_{+}(t+\tau)+p_{-}(t) p_{-}(t+\tau) \\
& \quad+2 \sqrt{p_{+}(t) p_{-}(t) p_{+}(t+\tau) p_{-}(t+\tau)}
\end{aligned}
$$

We have plotted, in Fig. 2, the relative entropy difference $S(t, \tau)$ and the fidelity difference $G(t, \tau)$ for this dynamical example. Here too, the non-Markovianity of the dynamics is clearly depicted by the negative values of $S(t, \tau)$ and $G(t, \tau)$.

\section{Example 3: Mixed separable state of two qubits}

Now we consider a separable mixed state of two qubits, $\rho_{S}(t=0)=\frac{1}{4}\left(I \otimes I+s_{x} \sigma_{1 x}+s_{y} \sigma_{1 y}+s_{z} \sigma_{1 z}+d \sigma_{1 y} \sigma_{2 x}\right)$
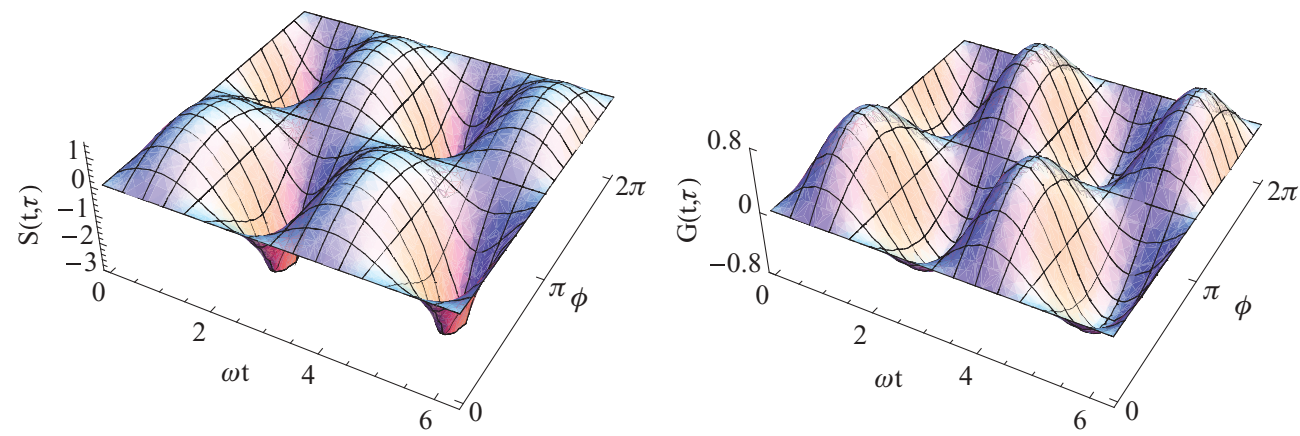

FIG. 1. (Color online) The relative entropy difference $S(t, \tau)$ and the fidelity difference $G(t, \tau)$ corresponding to the dynamical state (39), as a function of $\omega t$ and $\phi ; \omega \tau=\pi$. Negative regions of $S(t, \tau)$ and $G(t, \tau)$ point toward non-Markovian behavior. All quantities are dimensionless. 

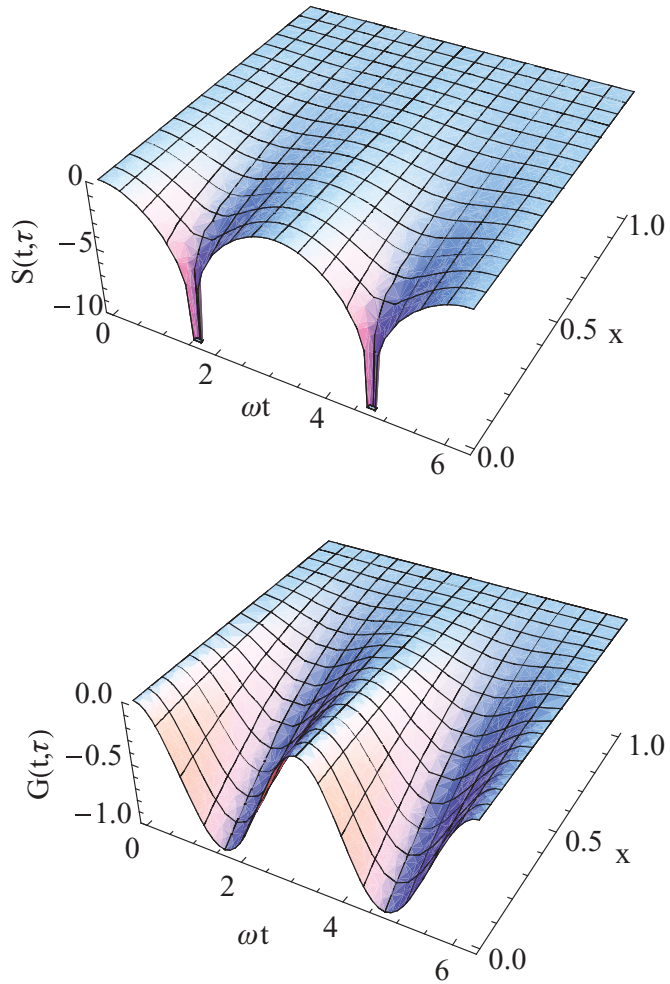

FIG. 2. (Color online) The relative entropy difference $S(t, \tau)$ and the fidelity difference $G(t, \tau)$ of the dynamical state (47), as a function of (dimensionless) time $\omega t$ and $x$; here, we have chosen $\omega \tau=\pi$. The functions $S(t, \tau)$ and $G(t, \tau)$ are negative in almost the entire region (except for $x=1$ ), reflecting the non-Markovianity of the underlying NCP dynamics. All quantities are dimensionless.
The initial state of the first qubit (system) is obtained to be

$$
\begin{aligned}
\rho_{S 1}(0) & =\operatorname{Tr}_{2}\left[\rho_{S}(0)\right] \\
& =\frac{1}{2}\left(\begin{array}{cc}
1+s_{z} & s_{x}-i s_{y} \\
s_{x}+i s_{y} & 1-s_{z}
\end{array}\right) .
\end{aligned}
$$

Open-system dynamics of the system qubit [corresponding to the joint unitary evolution (14)] leads to the dynamical state $\rho_{S 1}(t)$ as

$$
\begin{aligned}
\rho_{S 1}(t) & =\operatorname{Tr}_{2}\left[U(t) \rho_{S} U^{\dagger}(t)\right] \\
& =\frac{1}{2}\left(\begin{array}{cc}
1+s_{z} & s(t) \\
s^{*}(t) & 1-s_{z}
\end{array}\right),
\end{aligned}
$$

where we have denoted

$$
s(t)=\left(s_{x}-i s_{y}\right) \cos (\omega t)-d \sin (\omega t) .
$$

Note that in this example, we have the initial dynamical parameters $a_{1}=-\operatorname{Tr}\left[\rho_{S}(0) \sigma_{1 y} \sigma_{2 x}\right]=-d$ and $a_{2}=$ $\operatorname{Tr}\left[\rho_{S}(0) \sigma_{1 x} \sigma_{2 x}\right]=0$ and the dynamical state of the system (53) is equivalently obtained by transforming the initial density matrix (expressed as a column) through the $A$ matrix (23).

We evaluate the relative entropy $S\left[\rho_{S 1}(t) \| \rho_{S 1}(t+\tau)\right]$ of the state (53) to obtain

$$
\begin{aligned}
& S\left[\rho_{S 1}(t) \| \rho_{S 1}(t+\tau)\right] \\
& =\Omega^{(+)}(t) \ln \left\{\frac{\Omega^{(+)}(t)}{\left[\Omega^{(+)}(t+\tau)\right]^{\mu(t)}\left[\Omega^{(-)}(t+\tau)\right]^{\eta(t)}}\right\} \\
& \quad+\Omega^{(-)}(t) \ln \left\{\frac{\Omega^{(-)}(t)}{\left[\Omega^{(+)}(t+\tau)\right]^{\eta(t)}\left[\Omega^{(-)}(t+\tau)\right]^{\mu(t)}}\right\},
\end{aligned}
$$

where

$$
\begin{gathered}
\Omega^{( \pm)}(t)=\frac{1}{2}[1 \pm \zeta(t)], \quad \zeta(t)=\sqrt{s_{z}^{2}+\chi(t),} \quad \chi(t)=\left[s_{x} \cos (\omega t)-d \sin (\omega t)\right]^{2}+s_{y}^{2} \cos ^{2}(\omega t), \\
\mu(t)=\frac{1}{4 \zeta(t) \zeta(t+\tau)}\left\{\frac{\chi(t) \chi(t+\tau)}{\left[\zeta(t)-s_{z}\right]\left[\zeta(t+\tau)-s_{z}\right]}+\left[\zeta(t)-s_{z}\right]\left[\zeta(t+\tau)-s_{z}\right]+2 \mathcal{R}(t)\right\} \\
\eta(t)=\frac{1}{4 \zeta(t) \zeta(t+\tau)}\left\{\frac{\chi(t)\left[\zeta(t+\tau)-s_{z}\right]}{\zeta(t)-s_{z}}+\frac{\chi(t+\tau)\left[\zeta(t)-s_{z}\right]}{\zeta(t+\tau)-s_{z}}-2 \mathcal{R}(t)\right\} \\
\mathcal{R}(t)=\left(s_{x}^{2}+s_{y}^{2}\right) \cos (\omega t) \cos [\omega(t+\tau)]+d^{2} \sin (\omega t) \sin [\omega(t+\tau)]-d s_{x} \sin [\omega(2 t+\tau)] .
\end{gathered}
$$

The fidelity $F\left[\rho_{S 1}(t), \rho_{S 1}(t+\tau)\right]$ associated with the dynamical state (53) is found to be

$$
\begin{aligned}
F & {\left[\rho_{S 1}(t), \rho_{S 1}(t+\tau)\right] } \\
& =\frac{1}{2}\left\{1+s_{z}^{2}+\sqrt{\left[1-\zeta^{2}(t)\right]\left[1-\zeta^{2}(t+\tau)\right]}+\mathcal{R}(t)\right\},
\end{aligned}
$$

where $\zeta(t)$ and $\mathcal{R}(t)$ are defined in (56).

In Fig. 3, we depict the relative entropy difference $S(t, \tau)$ and the fidelity difference $G(t, \tau)$ of the state (53). The negative values of $S(t, \tau)$ and $G(t, \tau)$ demonstrate the non-Markovianity of the dynamical process in this example too.

We emphasize here that the negative values of the relative entropy difference and fidelity difference serve as qualitative reflections of deviation from a CP Markovian behavior-with no specific importance attached to the degree of negativity. Further, the positive regions in the figures do not indicate that the process is being Markovian in these regions-because our criteria offer only sufficient (not necessary) tests of non-Markovianity. It is also worth pointing out here that had we considered initially uncorrelated (or zero-discord) states, a CP dynamical map associated with the same unitary dynamics (14) would also be expected to exhibit nonMarkovianity [26]. In other words, it is not evident whether the departure from Markovianity is entirely from the NCP nature (resulting from initially correlated states) or from the built-in non-Markovianity associated with the dynamics. It 

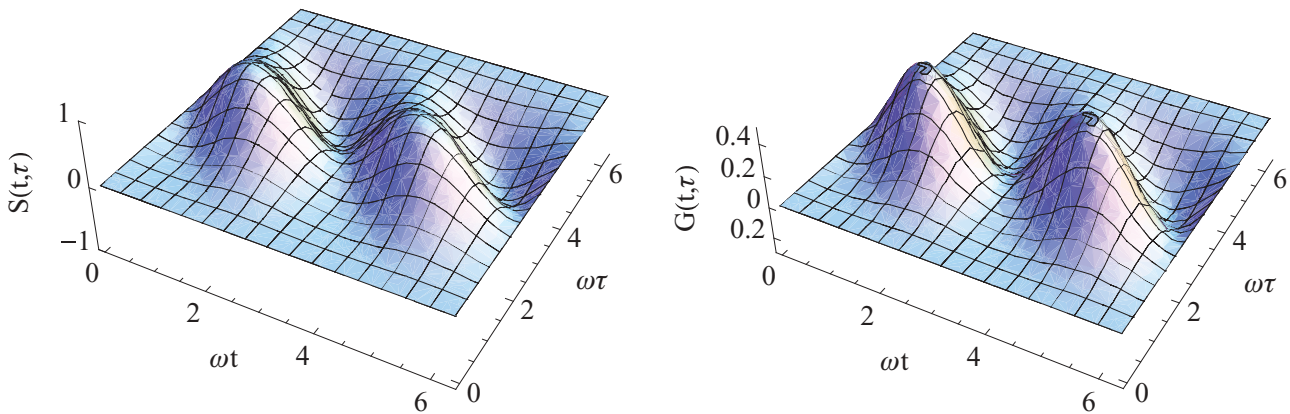

FIG. 3. (Color online) The relative entropy difference $S(t, \tau)$ and the fidelity difference $G(t, \tau)$ of the dynamical state (53), as a function of (dimensionless) time $\omega t$ and $\omega \tau$; here, we have chosen $s_{x}=s_{y}=s_{z}=d=1 / \sqrt{6}$. Negative fluctuations of the functions $S(t, \tau)$ and $G(t, \tau)$ reveal non-Markovianity. All quantities are dimensionless.

remains an open question to recognize distinct signatures of NCP non-Markovianity emerging exclusively due to initial system-environment correlations.

\section{SUMMARY}

While open-system evolution was formulated nearly five decades ago-with the introduction of dynamical $A$ and $B$ maps-by Sudarshan et al. [3,4], several interesting questions on the nature of dynamical maps have been raised recently [5-13]. It has been recognized that NCP dynamical maps make their presence felt in the reduced dynamics obtained from the joint unitary evolution, if the system and environment are in an initially correlated state [5-8]. A conceptual understanding of positive-but NCP dynamical maps-has thus been attracting increasing attention. In this paper, we have developed a canonical structure for the $A$ map and have shown that this canonical $A$ map offers an elegant approach to investigate whether the dynamics is CP or NCP. Manifestations of memory effects in $\mathrm{CP}$ open-system quantum evolution has been investigated in a previous work by some of us [23] and, here, we have focused on exploring the departure of CP Markovianity in a specifically chosen NCP dynamics [5] with initially correlated system-environment states. We have proposed a test to verify deviations from CP Markovianity, based on the relative entropy of the dynamical state of the system, which-together with an analogous characterization [23], in terms of the fidelity-is employed here to study prevalent memory effects in the NCP evolution. We have examined three different examples with diverse kinds of initial correlations: two qubits in (i) a pure entangled state, (ii) a Werner state (mixed two-qubit state which encompasses both entangled and separable states depending on a single real parameter $x$ ), and (iii) a separable state. All three dynamical examples considered here display non-Markovianity. However, it is not evident whether the reflections of non-Markovianity are essentially arising due to the NCP nature of the process (initially correlated states of qubits). This leaves open an important question: Are there any distinct signatures of non-Markovianity emerging entirely because of the NCP nature of the dynamical process?

\section{ACKNOWLEDGMENTS}

Sudha gratefully acknowledges financial support from the D.C. Pavate Foundation. She is also thankful to the local hospitality and facilities provided at Sidney Sussex College, Cambridge, UK.
[1] H.-P. Breuer and F. Petruccione, The Theory of Open Quantum Systems (Oxford University Press, Oxford, 2007).

[2] K. Kraus, States, Effects and Operations: Fundamental Notions of Quantum Theory, Vol. 190 of Lecture Notes in Physics (Spring-Verlag, New York, 1983).

[3] E. C. G. Sudarshan, P. Mathews, and J. Rau, Phys. Rev. 121, 920 (1961).

[4] T. F. Jordan and E. C. G. Sudarshan, J. Math. Phys. 2, 772 (1961).

[5] T. F. Jordan, A. Shaji, and E. C. G. Sudarshan, Phys. Rev. A 70, 052110 (2004).

[6] C. A. Rodríguez-Rosario, K. Modi, A. Kuah, A. Shaji, and E. C. G. Sudarshan, J. Phys. A 41, 205301 (2008).

[7] C. A. Rodríguez-Rosario and E. C. G. Sudarshan, e-print arXiv:0803.1183 [quant-ph].

[8] K. Modi and E. C. G. Sudarshan, Phys. Rev. A 81, 052119 (2010).
[9] P. Pechukas, Phys. Rev. Lett. 73, 1060 (1994).

[10] R. Alicki, Phys. Rev. Lett. 75, 3020 (1995); P. Pechukas, ibid. 75, 3021 (1995).

[11] P. Stelmachovic and V. Buzek, Phys. Rev. A 64, 062106 (2001).

[12] H. A. Carteret, D. R. Terno, and K. Życzkowski, Phys. Rev. A 77, 042113 (2008).

[13] A. Shabani and D. A. Lidar, Phys. Rev. Lett. 102, 100402 (2009).

[14] H. Ollivier and W. H. Zurek, Phys. Rev. Lett. 88, 017901 (2001).

[15] G. Lindblad, Commun. Math. Phys. 48, 119 (1976).

[16] V. Gorini, A. Kossakowski, and E. C. G. Sudarshan, J. Math. Phys. 17, 821 (1976).

[17] S. Daffer, K. Wódkiewicz, J. D. Cresser, and J. K. McIver, Phys. Rev. A 70, 010304 (2004).

[18] H.-P. Breuer, Phys. Rev. A 70, 012106 (2004). 
[19] D. Chrusćinśki, and A. Kossakowski, Phys. Rev. Lett. 104, 070406 (2010); D. Chrusćinśki, and A. Kossakowski, and S. Pascazio, Phys. Rev. A 81, 032101 (2010).

[20] M. M. Wolf, J. Eisert, T. S. Cubitt, and J. I. Cirac, Phys. Rev. Lett. 101, 150402 (2008).

[21] H.-P. Breuer, E.-M. Laine, and J. Piilo, Phys. Rev. Lett. 103, 210401 (2009); E.-M. Laine, J. Piilo, and H.-P. Breuer, Phys. Rev. A 81, 062115 (2010).

[22] A. Rivas, S. F. Huelga, and M. B. Plenio, Phys. Rev. Lett. 105, 050403 (2010).

[23] A. K. Rajagopal, A. R. Usha Devi, and R. W. Rendell, Phys. Rev. A 82, 042107 (2010).

[24] M. D. Choi, Can. J. Math. 24, 520 (1972); Linear Algebra Appl. 10, 285 (1975).
[25] The eigenvalues of $\mathcal{A}$ are the same as that of the $B$ matrix considered in Ref. [5].

[26] The eigenvalues (27) reduce to $\{1+\cos (\omega t), 0,1-\cos (\omega t), 0\}$ when $a_{1}=a_{2}=0$ (which corresponds to an initially uncorrelated state) and the associated dynamical map is CP. It may be readily verified-based on the time dependence of the associated Kraus operators (see [23]) of this CP dynamical map with uncorrelated initial state-that the dynamics is non-Markovian.

[27] M. A. Nielsen and I. L. Chaung, Quantum Computation and Quantum Information (Cambridge University Press, Cambridge, 2002).

[28] M. B. Ruskai, J. Math. Phys. 43, 4358 (2002).

[29] R. Jozsa, J. Mod. Opt. 41, 2315 (1994). 